\title{
AN INTENSITY DISTRIBUTION OF BRIGHT POINTS OBSERVED ON A CN SPECTROHELIOGRAM
}

\author{
(Research Note)
}

\author{
BRUCE GILLESPIE \\ The Department of Astronomy, The University of Michigan, Ann Arbor, Michigan, and \\ Kitt Peak National Observatory,* Tucson, Arizona
}

(Received 24 September, 1971)

Intimate spatial and quantitative relations have recently been noted between the photospheric network as observed in temperature-sensitive lines and the local magnetic fields (Chapman and Sheeley, 1968; Sheeley and Engvold, 1970). This note is a report of photometry of bright photospheric network 'points' observed on a spectroheliogram taken under optimal seeing conditions.

On the morning of 4 July 1970, a spectroheliogram was taken in the bandhead of CN $\lambda 3883 \AA$ with the 15 -ft spectroheliograph fed with the $38 \mathrm{~cm}$ image of the east auxiliary telescope of the McMath Solar Telescope. On the plate is a strip in which one can resolve features of approximately $0.5 \mathrm{arcsec}$ in diameter. The entrance and exit slits were $75 \mu$ wide, corresponding to 0.38 arc sec at the solar image, and to a bandpass of $0.087 \AA$ in the third order of the grating. By good fortune, this belt of good seeing contains the image of a well-developed active region, including a large sunspot and its associated plage.

The peak photographic density of 109 discrete bright 'points' just outside of the edge of the sunspot penumbra was measured (Figure 1) with a square aperture $45 \mu$ on a side. The majority of the points had apparent linear extents of five to ten times the linear size of the scanning aperture. Relative plate density was calibrated in terms of relative intensity by means of an image on the plate of a photoelectrically standardized stepwedge.

Using the characteristic curve of the emulsion, the peak intensity of each of the 109 bright points was obtained, relative to the average intensity of the nearby quiet non-network region. The measuring process of the points was then repeated in its entirety. The small differences between the two sets of peak intensities were used to compute the errors of the measurements.

Some statistical parameters of the observed distribution were calculated and are listed in Table I.

Figure 2 shows the $\Delta I / I$ distribution fitted with a normal curve. Selection bias precluded the observation of points with intensities of less than $\Delta I / I=0.40$. A number of these points do exist, but they are so well hidden in the background intensity

* Operated by the Association of Universities for Research in Astronomy, Inc., under contract with the National Science Foundation. 


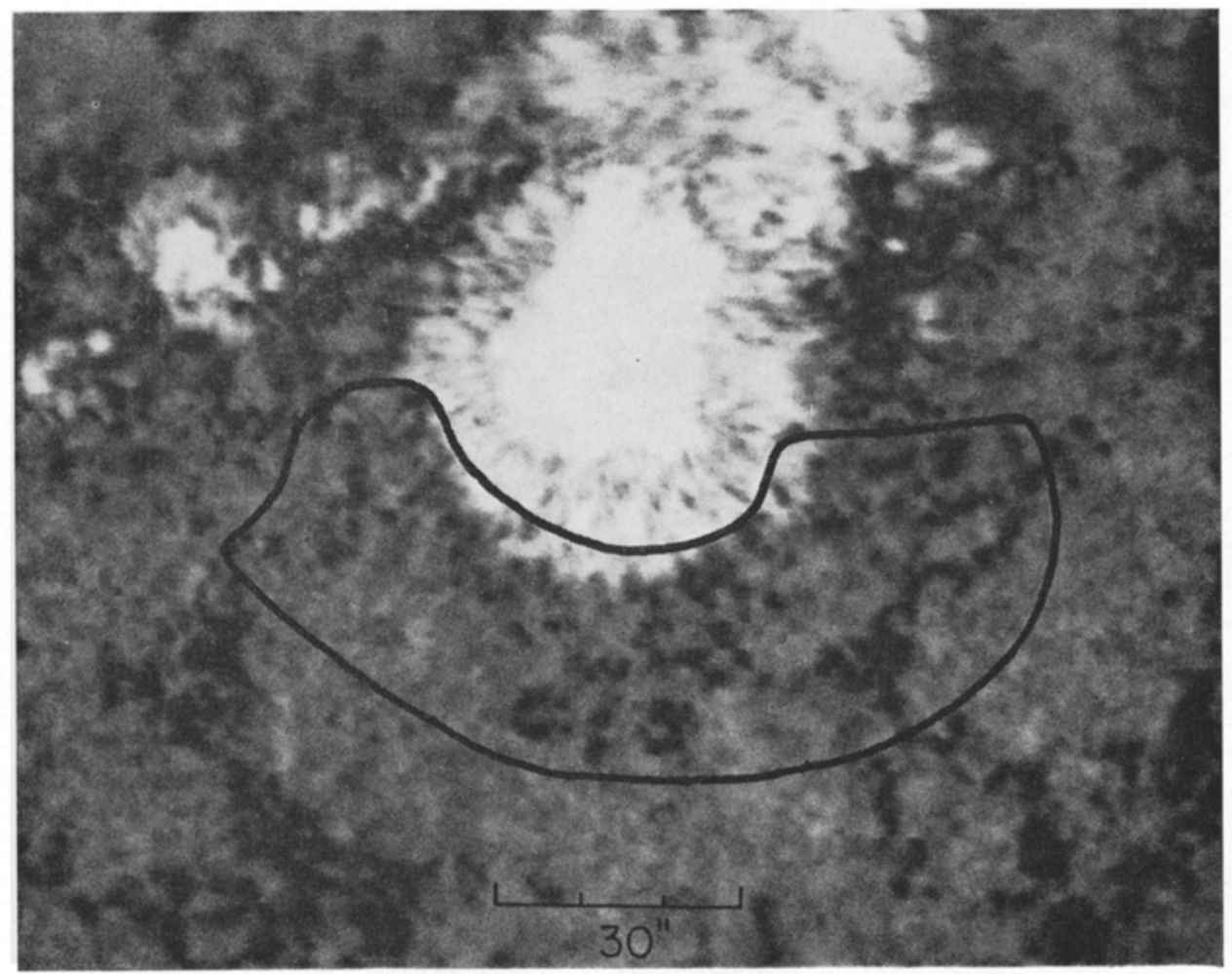

Fig. 1. A high resolution $\mathrm{CN}$ spectroheliogram showing part of the active region around $\mathrm{Mt}$. Wilson Sunspot No. 17995. The exposure was taken at 8:35 MST on the morning of 4 July 1970. This negative enlargement shows the bright 'points' as darker than average features. Within the enclosed area, 109 bright points were measured to build the intensity distribution of Figure 2.

TABLE I

Statistical parameters of the $\mathrm{CN}$ bright point $\Delta I / I$ distribution

Sample points: 109

Observed limits: $\Delta I / I=0.40$ to 1.43 (lower limit due to selection effects)

Mean error of a single observation: $\Delta I / I= \pm 0.01$

Mean peak intensity: $\Delta I / I=0.79$

Standard deviation: $\Delta I / I=0.22$

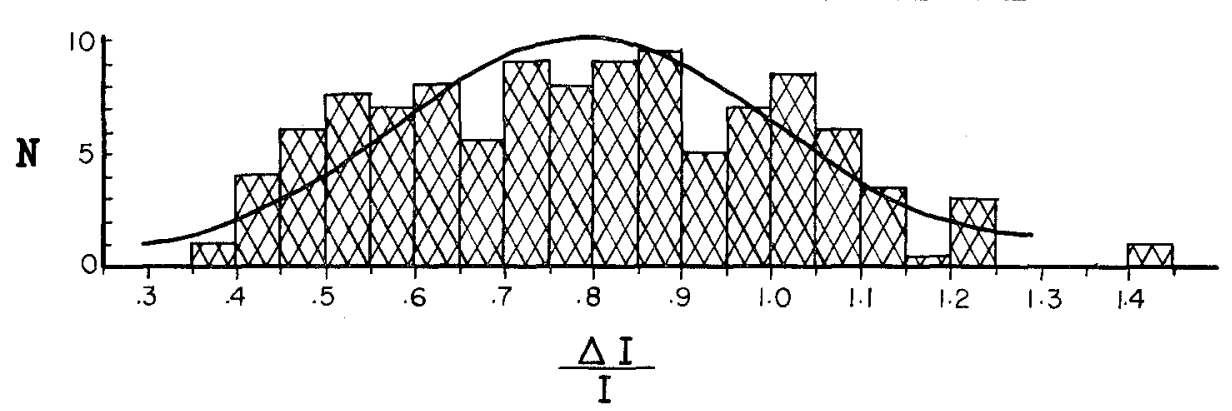

Fig. 2. The observed intensity distribution with a fitted normal curve. 
variations that only by time-averaging several consecutive spectroheliograms can they be distinctly observed (Sheeley, 1971).

Seeing and instrumental smearing affect the results, making the measured peak intensities lower than the true values. While the average diameter of the points was a little in excess of $1.5 \mathrm{arcsec}$, an upper limit on the size of the seeing disk is about one third that size, as estimated from measuring the smallest discernable features. However, for most points, this central region was rather large and uniform in photographic density, which suggests that it was not appreciably smeared. The characteristic curve of the emulsion showed that in every case the images of the bright points had not saturated. It might therefore be fair to suppose that the large breadth and flatness of the observed distribution is for the most part real, rather than instrumental.

\section{Acknowledgements}

I am indebted to Dr Helen Dodson Prince and Mr Clifford Bennett for their assistance and the use of the facilities of the McMath-Hulbert Observatory. I wish to acknowledge the help of Drs Neil Sheeley, Jr. and Richard Teske.

\section{References}

Chapman, G. A. and Sheeley, Jr., N. R.: 1968, Solar Phys. 5, 442.

Sheeley, Jr., N. R.: 1969, Solar Phys. 9, 347.

Sheeley, Jr., N. R.: 1971, Solar Phys. $20,19$.

Sheeley, Jr., N. R. and Engvold, O.: 1970, Solar Phys. 12, 69. 